\title{
Hyperosmotic stress induces cisplatin sensitivity in ovarian cancer cells by stimulating aquaporin-5 expression
}

\author{
XUEJUN CHEN ${ }^{*}$, CHUNXIA ZHOU* ${ }^{*}$ CHUNXIAO YAN, JIONG MA and WEI ZHENG \\ Department of Gynecology, Second Affiliated Hospital, College of Medicine, \\ Zhejiang University, Hangzhou, Zhejiang 310009, P.R. China
}

Received October 10, 2014; Accepted September 18, 2015

DOI: $10.3892 / \mathrm{etm} .2015 .2833$

\begin{abstract}
Aquaporins (AQPs) are important mediators of water permeability and are closely associated with tumor cell proliferation, migration, angiogenesis and chemoresistance. Moreover, the chemosensitivity of tumor cells to cisplatin (CDDP) is potentially affected by osmotic pressure. The present study was undertaken to determine whether hyperosmosis regulates ovarian cancer cell sensitivity to CDDP in vitro and to explore whether this is associated with AQP expression. The hyperosmotic stress was induced by $\mathrm{D}$-sorbitol. $3 \mathrm{AO}$ ovarian cancer cells were treated with different concentrations of hypertonic medium and/or CDDP for various times, followed by measuring the inhibition rate of cell proliferation using an MTT assay. In addition, AQP expression in response to osmotic pressure and/or CDDP was measured by reverse transcription-quantitative polymerase chain reaction and western blotting. Cell proliferation in response to hypertonic stress was also measured when AQP5 was knocked down by small interfering (si)RNA. 3AO cell proliferation was inhibited by hyperosmotic stress, while the expression of AQP5, but not that of AQP1, AQP3 or AQP9, was increased in a dose- and time-dependent manner in hypertonic sorbitol-containing medium. When AQP5 was silenced by siRNA, cells were susceptible to hypertonic stress. MTT analyses showed that the inhibition of cell proliferation by a low dose of CDDP increased significantly with exposure to a hyperosmotic stimulus, and this effect was reduced when a high dose of CDDP was used. AQP5 expression was induced by a low dose of CDDP, but was reduced by a high dose of CDDP. However, hyperosmosis enhanced AQP5 mRNA expression at
\end{abstract}

Correspondence to: Professor Wei Zheng, Department of Gynecology, Second Affiliated Hospital, College of Medicine, Zhejiang University, 88 Jiefang Road, Hangzhou, Zhejiang 310009, P.R. China

E-mail: honoredzheng@126.com

*Contributed equally

Key words: ovarian cancer, osmotic pressure, cisplatin sensitivity, aquaporin 5 every dose of CDDP tested, compared with isotonic medium. With prolonged treatment time, AQP5 expression was reduced by CDDP in hypertonic and isotonic culture medium. Thus, the effects of hyperosmosis on cell sensitivity to CDDP were associated with AQP5 expression. These results suggest that AQP5 expression in ovarian cancer cells is induced by hypertonic medium, and that the sensitivity of ovarian cancer cells to CDDP can be regulated by hyperosmosis associated with AQP5 expression.

\section{Introduction}

There are few specific early symptoms or sensitive biomarkers for the screening of ovarian cancer. Thus, the majority of patients with ovarian cancer are not diagnosed until stage III-IV when they are no longer eligible for the most effective surgical interventions. Platinum-based chemotherapy has been a critical treatment for ovarian cancer since the late 1970 s and has improved overall survival significantly (1). However, initial chemotherapy resistance and platinum-resistant relapse occur frequently, causing increased mortality due to progressive disease (2). It is therefore imperative to find an approach to overcome resistance to cisplatin (CDDP).

The aquaporins (AQPs) are a family of small transmembrane proteins that primarily facilitate the rapid, passive movement of water or small sugar alcohol molecules, such as glycerol, across cell plasma membranes and are essential for cellular water homeostasis. AQPs are ubiquitously expressed in all types of organisms from bacteria to plants, insects and mammals $(3,4)$. Amino acid sequence and molecular function have been used to categorize AQPs into three distinct subgroups: Classical aquaporins, aquaglyceroporins and unorthodox aquaporins (5-8). The first group includes AQP0, AQP1, AQP2, AQP4 and AQP5, which have highly selective permeability to water but not other molecules. The aquaglyceroporin group includes AQP3, AQP7, AQP9 and AQP10, which are permeable to water, as well as glycerol, urea and other small non-electrolytes. AQP6, AQP8, AQP11 and AQP12 are classified as unorthodox aquaporins, and their functions remain under investigation (8).

It has been revealed that AQPs are unusually expressed in numerous kinds of human cancers. According to the review by Ribatti et al, AQP1, AQP3, AQP4, AQP5, AQP8 and AQP9 are closely associated with various kinds of tumors (9). In 
ovarian tumors, the localization and expression patterns of AQP1-9 have been investigated using immunohistochemistry; AQP1, AQP3, AQP5, AQP6, AQP8 and AQP9 were identified in epithelial ovarian cancer, while AQP1, AQP5 and AQP9 were significantly overexpressed in malignant and borderline tumors compared with benign tumors and normal ovarian tissue (10). Another study revealed that the expression of AQP1 was not significantly associated with the clinicopathological stage in serous epithelial ovarian cancer (11), although AQP1 expression was associated with ascites, intratumoral microvessel density (IMD) and clinicopathological variables. AQP5 is highly expressed in lymph node metastasis cases and in abundant ascites, and previous studies determined that AQP9 expression correlated with the degree of histological malignancy $(10,12,13)$. It has also been reported that AQP3 facilitates ovarian cancer cell migration and correlates with epidermal growth factor (EGF)-induced cell metastasis (14). A previous study by our group demonstrated that CDDP downregulates AQP5 in a concentration-dependent manner in CAOV3 cells, and that nuclear factor $(\mathrm{NF})-\kappa \mathrm{B}$ is involved in AQP5 regulation (15). Epigallocatechin gallate, which inhibits proliferation and induces apoptosis of SKOV3 cells, decreased the expression of AQP5, suggesting a possible association between ovarian cancer cell proliferation and AQP5 protein expression (16). These studies indicate that AQP might be a new therapeutic target for ovarian cancer.

AQPs are involved in the fluidity and integrity of cell membranes, angiogenesis, cell migration and cell volume regulation $(9,17)$. It has been recognized that water transport across cytomembranes could be modified by osmotic stress through AQP proteins, and that an increase in AQP3 or AQP9 expression is associated with increased chemoresistance to arsenite in melanoma, lung cancer, primary cultured chorion and amnion cells (18-20). AQP expression has also been found to affect chemosensitivity in ovarian carcinoma (21), which may be associated with osmosis.

To demonstrate whether chemotherapeutic drug sensitivity and resistance are affected by water permeability and AQP expression in ovarian cancer, the effects of extracellular hyperosmotic stress on AQP expression and sensitivity to CDDP were investigated in the present study.

\section{Materials and methods}

Cell culture and reagents. Ovarian cancer cell line $3 \mathrm{AO}$ was obtained from the Institute of Cancer Research, Chinese Academy of Medical Sciences (Beijing, China). 3AO cells were cultured in Dulbecco's modified Eagle's medium (DMEM; Gibco BRL, Gaithersburg, MD, USA) supplemented with $15 \%$ fetal bovine serum and maintained at $37^{\circ} \mathrm{C}$ and $5 \% \mathrm{CO}_{2}$ in a humid environment. For osmotic stress, hyperosmotic medium was made by adding various concentrations of D-sorbitol (Sigma-Aldrich, St. Louis, MO, USA) to regular DMEM for different times. Standard DMEM was used as isosmotic media.

Cell growth and inhibition rate assay. Cells were treated with the following solutions: i) DMEM medium alone (the control group); ii) $0.625,1.25,2.5,5,10$ or $20 \mu \mathrm{g} / \mathrm{ml} \mathrm{CDDP}$ (Sigma-Aldrich) for 24,48 or $72 \mathrm{~h}$; iii) 100, 200, 300, 400, 500,600 or $800 \mathrm{mM}$ D-sorbitol for 24,48 or $72 \mathrm{~h}$; iv) $200 \mathrm{mM}$
D-sorbitol and $0.625,1.25,2.5,5,10$ or $20 \mu \mathrm{g} / \mathrm{ml} \mathrm{CDDP}$ for 24 , 48 or $72 \mathrm{~h}$. Cell growth and inhibition rate was measured by 3-(4,5-dimethylthiazol-2-yl)-2,5-diphenyltetrazolium bromide (MTT) assay.

$3 \mathrm{AO}$ cells were seeded in 96 -well plates $(5,000$ cells per well) for $24 \mathrm{~h}$ and exposed to various concentrations of CDDP or D-sorbitol for 24-72 h. Following treatment, the cells were incubated with $10 \mu 15 \mathrm{mg} / \mathrm{ml}$ MTT (Sigma-Aldrich) $4 \mathrm{~h}$ at $37^{\circ} \mathrm{C}$ in the dark. The formazan crystals were lysed with $150 \mu$ l dimethyl sulfoxide (DMSO; Sigma-Aldrich) for $10 \mathrm{~min}$. Absorbance values (optical density; OD) at a wavelength of $490 \mathrm{~nm}$ were obtained using a microplate reader (model 680: Bio-Rad Laboratories, Inc., Hercules, CA, USA). Wells containing cells in DMEM and wells containing no cells served as the normal control and background control, respectively. To convert OD values to a growth inhibition rate, the following equation was used: Inhibition rate $=(O D$ of control $-O D$ of test concentration)/(OD of control - OD of cell-free wells). Each concentration was evaluated in 3-5 repeated wells, and every assay was performed at $\geq 3$ times.

RNA isolation and reverse transcription-quantitative polymerase chain reaction $(R T-q P C R)$. The expression of AQP5 mRNA was examined by RT-qPCR. Total RNA was isolated from $3 \mathrm{AO}$ cells with TRIzol reagent (Thermo Fisher Scientific, Inc., Waltham, MA, USA). RNA was quantified using a NanoDrop ND-1000 spectrophotometer (Thermo Fisher Scientific, Inc.). Those samples whose $\mathrm{A}_{260} / \mathrm{A}_{280}$ ratios were 1.8-2.0 were used for further analysis. Then, $1 \mu \mathrm{g}$ RNA was used for reverse transcription conducted using a PrimeScript $^{\mathrm{TM}}$ RT reagent kit with gDNA eraser (Takara Bio, Tokyo, Japan) according to the instructions provided by the manufacturer. qPCR was carried out in a $20-\mu 1$ reaction volume containing $1 \mu \mathrm{l}$ cDNA with SYBR Premix Ex $\mathrm{Taq}^{\mathrm{TM}}$ (Takara Bio) using an Applied Biosystems StepOne Fast Real-Time PCR system (Thermo Fisher Scientific, Inc.). The sequences of the primers used were as follows: AQP1 (NM_198098), sense: 5'-ATCCTCTCAGGCATCACCTC-3' and antisense: 5'-GGTAGTAGCCAGCACGCATA-3'; AQP3 (NM_004925), sense: 5'-CAGTGGGACGTGTTTCTGTC-3' and antisense: 5'-CCCGGATCCCTAAGACTGTA-3'; AQP5 (NM_001651), sense: 5'-CTGTCCATTGGCCTGTCTGTC-3' and antisense 5'-GGCTCATACGTGCCTTTGATG-3'; AQP9 (NM_020980), sense: 5'-CCTGAAACAGCCTTC TCTCC-3' and antisense: 5'-AAACCACCCAAATGGGAC TA-3'; glyceraldehyde 3-phosphate dehydrogenase (GAPDH), sense: 5'-CATCAATGGAAATCCCATCA-3' and antisense: 5'-TTCTCCATGGTGGTGAAGAC-3'.

The specificity of primers was tested by running a regular PCR followed by agarose gel electrophoresis. The conditions of the amplification process were: $95^{\circ} \mathrm{C}$ for $30 \mathrm{sec}, 95^{\circ} \mathrm{C}$ for $5 \mathrm{sec}$ and $60^{\circ} \mathrm{C}$ for $30 \mathrm{sec}$, for 45 cycles. Each cDNA sample was analyzed in triplicate, and GAPDH primer was included in every plate as an internal control. Melting curve data were collected to assure PCR specificity. All qPCRs were performed in more than triplicate. Relative quantification of AQP5 mRNA expression was calculated using the $2^{-\Delta \Delta \mathrm{Cq}}$ method.

Western blot analysis. Cells with or without treatments were washed with cold phosphate-buffered saline and harvested by 
scraping in radioimmunoprecipitation assay (RIPA) buffer containing protease inhibitor cocktail (Sigma-Aldrich). Total protein was extracted with RIPA buffer containing protease inhibitor for $30 \mathrm{~min}$ on ice. After centrifugation at $13,000 \mathrm{x} \mathrm{g}$ for $15 \mathrm{~min}$ at $4^{\circ} \mathrm{C}$, protein concentrations were determined by Bio-Rad protein assay (Bio-Rad Laboratories, Inc.). A 40- $\mu \mathrm{g}$ quantity of total protein was subjected to $10 \%$ sodium dodecyl sulfate-polyacrylamide gel electrophoresis (SDS-PAGE) and then transferred onto a polyvinylidene (PVDF) membrane (Millipore, Bedford, MA, USA). After being blocked for $1 \mathrm{~h}$ with 5\% non-fat milk in Tris-buffered saline and Tween 20 (TBS-T) at room temperature, the membranes were incubated with rabbit polyclonal anti-AQP5 (1:200; BA2200-2; Boster Biological Technology, Wuhan, China) or mouse monoclonal anti-GAPDH (1:5,000; TA-08; Zhongshan Golden Bridge Biotechnology Co., Ltd., Beijing, China) primary antibody at $4^{\circ} \mathrm{C}$ overnight. The membranes were then washed three times for 10 min each with TBS-T, and incubated with a 1:5,000 dilution of goat anti-rabbit (ZB-5301) or goat anti-mouse (ZB-5305) secondary antibody labeled with horseradish peroxidase (Zhongshan Golden Bridge Biotechnology Co., Ltd.) for $1 \mathrm{~h}$ at room temperature. Antibody binding was detected using an enhanced chemiluminescence (ECL; Millipore) detection system following the manufacturer's instructions.

Small interfering (si)RNA transfection. A lentiviral vector (LV) expressing AQP5-siRNA and equipped with green fluorescence protein (GFP) and puromycin acetyltransferase protein (PACP) was constructed by Shanghai GenePharma Co., Ltd. (Shanghai, China). The provided control siRNA was used as a negative control (mock). 3AO cells were transfected with 30 multiplicity of infection (MOI) of AQP5-siRNA in the presence of $6 \mathrm{ng} / \mathrm{ml}$ Polybrene (Sigma-Aldrich). The medium containing the siRNA was replaced with fresh medium after $24 \mathrm{~h}$ transfection. Green fluorescence was observed after $48 \mathrm{~h}$ incubation. Then, cells were screened in medium containing puromycin ( $3 \mu \mathrm{g} / \mathrm{ml}$; Sigma-Aldrich) for 3 weeks. Following the selection of transfected $3 \mathrm{AO}$ cells, in order to test the effects of AQP5-siRNA, AQP5 expression was detected by RT-PCR and western blotting.

Statistical analysis. The data are expressed as mean \pm standard deviation (SD). Values were analyzed by one-way analysis of variance (ANOVA) and Student's unpaired t-test. $\mathrm{P}<0.05$ was considered to indicate a statistically significant result.

\section{Results}

Effects of hyperosmotic stress on the proliferation of ovarian cancer cells. $3 \mathrm{AO}$ cells were incubated with various concentrations of D-sorbitol, representing different osmotic pressures, for various times, and the inhibition of cell proliferation was measured by MTT assay. Results showed that $3 \mathrm{AO}$ cell proliferation was reduced in a dose- and time-dependent manner in hypertonic culture medium. When $3 \mathrm{AO}$ cells were treated with $200 \mathrm{mM}$ D-sorbitol for 24,48 or $72 \mathrm{~h}$, the inhibition rate of cell proliferation was 10.08, 20.52 and 31.63\%, respectively (Fig. 1).

Effect of hyperosmotic stress on the mRNA expression of $A Q P s$ in ovarian cancer cells. AQP expression is affected by

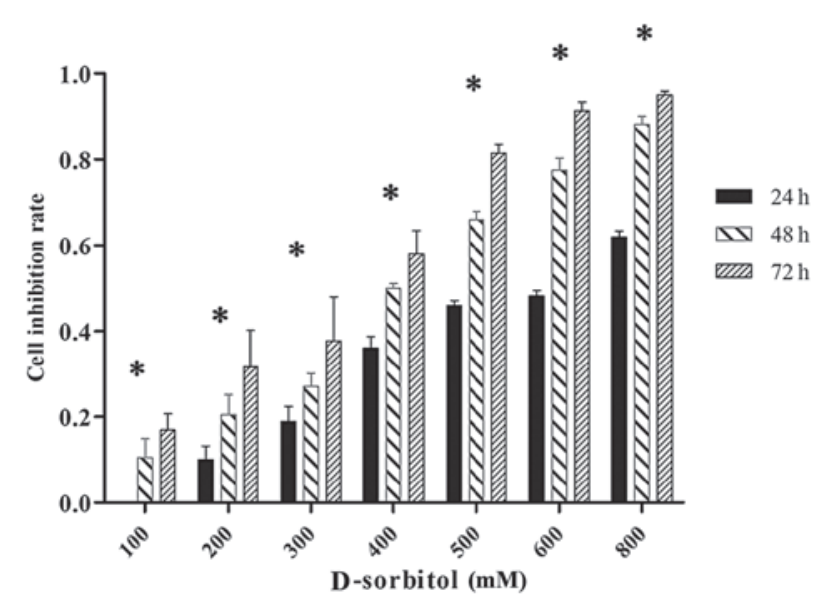

Figure 1. Cell inhibition rate induced by hypertonic medium. Ovarian cancer cell proliferation was inhibited by hyperosmotic pressure in a concentrationand time-dependent manner. ${ }^{*} \mathrm{P}<0.05$, dose group vs time group at mean time.

hyperosmotic stress in several cell types. To examine the AQP expression in response to extracellular hyperosmotic stress in ovarian cancer, the mRNA expression levels of various AQPs were measured by RT-qPCR in $3 \mathrm{AO}$ cells that were incubated with $50,100,200,300$ or $400 \mathrm{mM}$ D-sorbitol (hypertonic medium) for 12, 24 or $48 \mathrm{~h}$. It was found that AQP5 mRNA expression levels increased significantly when the cells were treated with hyperosmotic medium for $24 \mathrm{~h}$. However, AQP5 mRNA expression peaked at the $200 \mathrm{mM}$ concentration of D-sorbitol; at higher concentrations of D-sorbitol, the expression of AQP5 was reduced, yet it remained higher than that in control cultures $(\mathrm{P}<0.05$; Fig. $2 \mathrm{~A})$. By contrast, the expression levels of AQP1, AQP3 and AQP9 mRNA were only slightly elevated by hypertonic sorbitol-containing medium (Fig. 2D-F).

When cells were incubated with hypertonic medium for $48 \mathrm{~h}$, the expression levels of AQP1, AQP3, AQP9 mRNA were still only slightly increased (Fig. 2G-I); however, AQP5 expression was increased continuously and markedly $(\mathrm{P}<0.05$; Fig. 2B) as the osmotic pressure increased. To examine the time course of AQP5 expression, 3AO cells were treated with $200 \mathrm{mM}$ D-sorbitol for 12, 24 and $48 \mathrm{~h}$, followed by the analysis of AQP5 mRNA expression levels using RT-qPCR. The outcomes showed that AQP5 mRNA expression was increased in a time-dependent manner in hypertonic medium $(\mathrm{P}<0.05$; Fig. 2C). Western blot analysis indicated that post-transcriptional AQP5 expression was induced in a similar manner to transcriptional level expression by hyperosmotic pressure (Fig. 2J).

Effects of AQP5 silencing on the response to hyperosmotic stress in $3 A O$ cells. To determine the contribution of AQP5 to hyperosmotic stress, $3 \mathrm{AO}$ cells were transfected with LV-siRNA-AQP5 or LV-siRNA-mock siRNA constructs. Transfection efficiency was confirmed by RT-PCR and western blotting (Fig. 3A and B). MTT assays were performed on transfected cells incubated in D-sorbitol-containing medium for $24 \mathrm{~h}$. The inhibition rate of cell proliferation was significantly increased in cells transfected with AQP5 siRNA compared with that in mock-transfected controls in response 
A

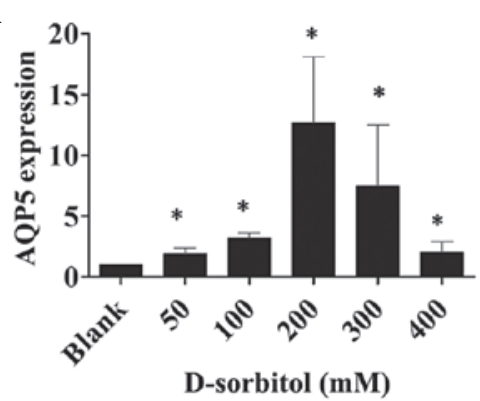

D

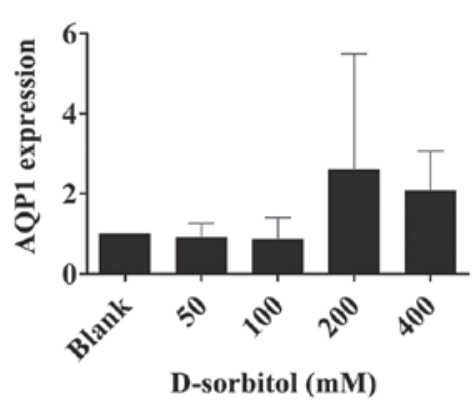

G

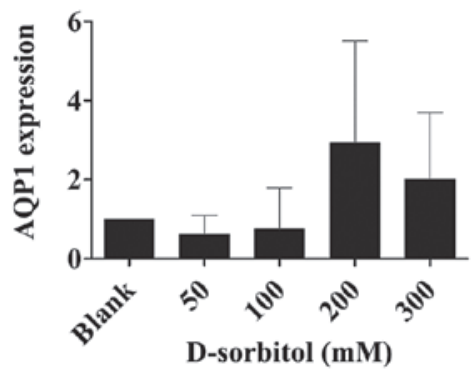

B

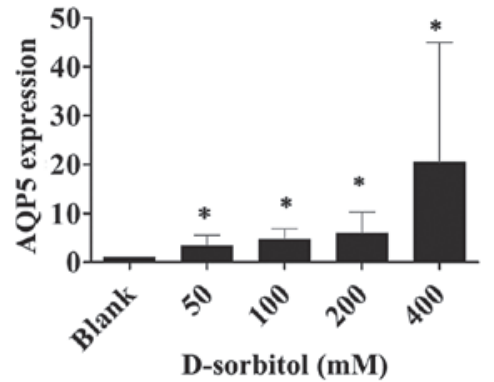

$\mathbf{E}$

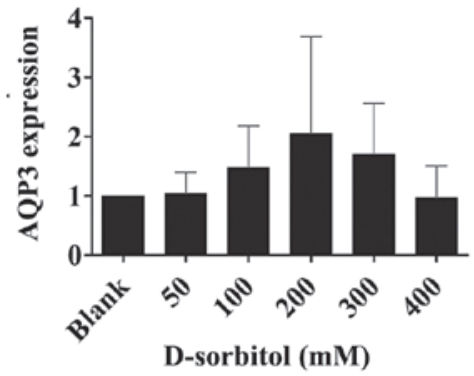

$\mathbf{H}$

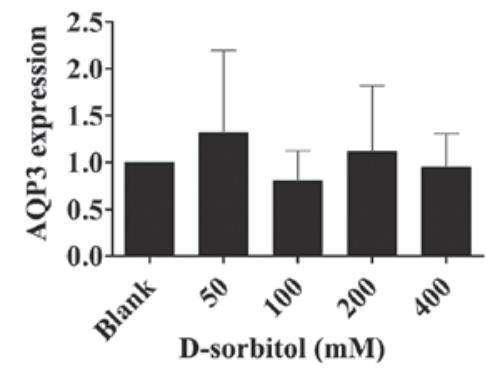

C

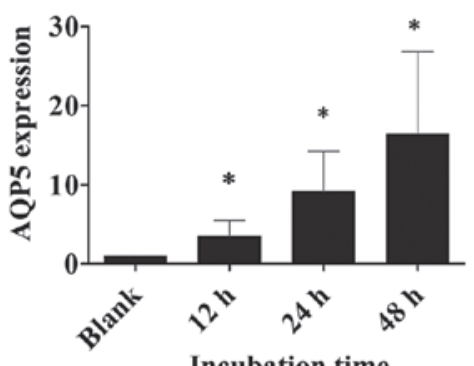

$\mathbf{F}$

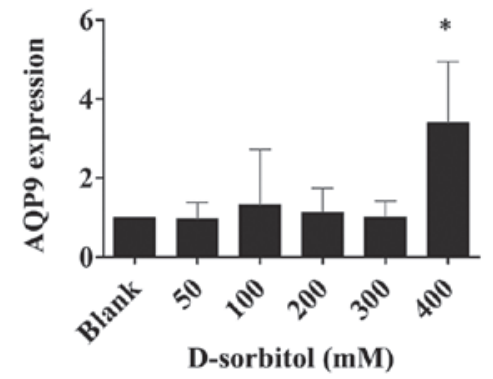

I

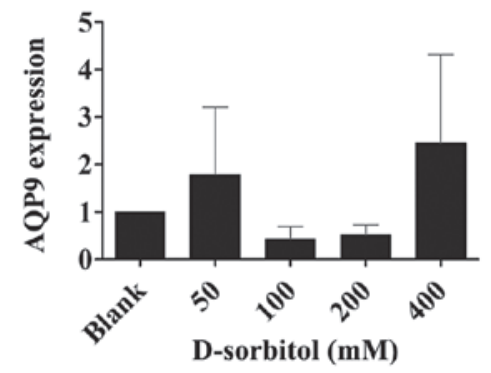

$\mathbf{J}$

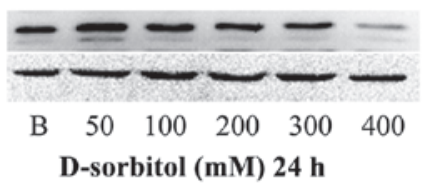

AQP5 $(28 \mathrm{kDa})$

GAPDH $(37 \mathrm{kDa})$

Figure 2. Expression of various AQPs in 3AO ovarian cancer cells in response to hypertonic D-sorbitol medium. AQP5 expression measured by RT-qPCR in $3 \mathrm{AO}$ cells that were incubated in isotonic (blank) and hypertonic medium (50-400 mM D-sorbitol) for (A) $24 \mathrm{~h}$ or (B) $48 \mathrm{~h}$. (C) AQP5 mRNA expression measured by qRT-PCR in 3AO cells that were treated with $200 \mathrm{mM}$ D-sorbitol for 12, 24 and $48 \mathrm{~h}$. mRNA expression of (D and G) AQP1, (E and H) AQP3 and (F and I) AQP9 detected by qRT-PCR in 3AO cells treated with hypertonic D-sorbitol (50-400 mM) medium and normal medium (blank) for (D-F) $24 \mathrm{~h}$ and (G-I) 48 h. (J) AQP5 protein expression determined by western blotting when the cells were cultured with 50-400 mM D-sorbitol medium or regular medium (blank) for $24 \mathrm{~h}$, GAPDH was used as an intrinsic control. "P<0.05 vs. blank. AQP, aquaporin; RT-qPCR, reverse transcription-quantitative polymerase chain reaction; B, blank; GAPDH, glyceraldehyde 3-phosphate dehydrogenase.

to hypertonic medium (Fig. 3C). The attenuated reactivity of ovarian cancer cells to hyperosmotic pressure increased incrementally with escalating osmotic concentrations in the cells transfected with AQP5 siRNA, which indicated that AQP5 expression facilitated protective mechanisms in response to hypertonic conditions.

Effect of hyperosmotic stress on cell sensitivity to CDDP. The addition of $200 \mathrm{mM}$ D-sorbitol to the extracellular environment induced hyperosmotic pressure, which elevated AQP5 expression levels and caused slight cytotoxicity (Figs. 1 and 2C). CDDP had variable inhibitory effects on cell proliferation in response to either hypertonic or isotonic conditions. MTT results showed that the inhibition rate of cell proliferation induced by CDDP was increased in extracellular hyperosmotic medium when the CDDP concentration was $<2.5 \mu \mathrm{g} / \mathrm{ml}$ and decreased in hyperosmotic medium with CDDP concentrations of 5-20 $\mu \mathrm{g} / \mathrm{ml}$ (Fig. 4). These results were more pronounced when the incubation time was prolonged to 48 or $72 \mathrm{~h}$. Even though hyperosmosis or CDDP alone can lead to cellular damage, together they exerted an additive effect on cytotoxicity for CDDP concentrations of 0.625 and $1.25 \mu \mathrm{g} / \mathrm{ml}$. Furthermore, it was found that the decreased sensitivity to CDDP caused by extracellular hyperosmosis at 5 and $10 \mu \mathrm{g} / \mathrm{ml}$ CDDP was significant. In general, the sensitivity of ovarian cancer cells to CDDP was changed by exposure to 
A

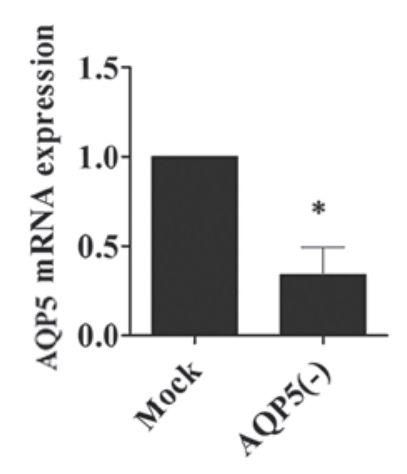

B

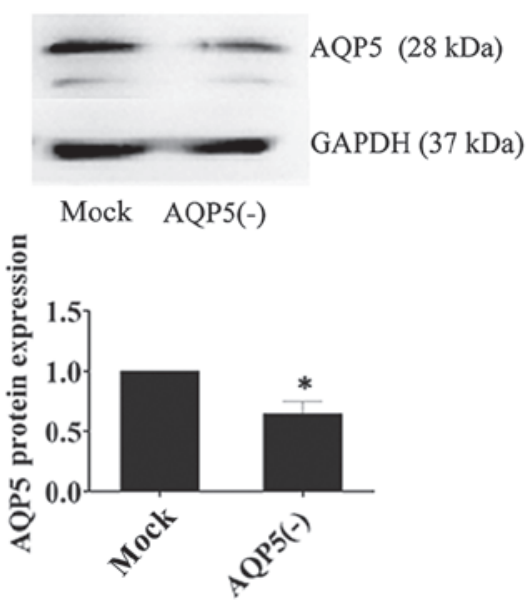

C

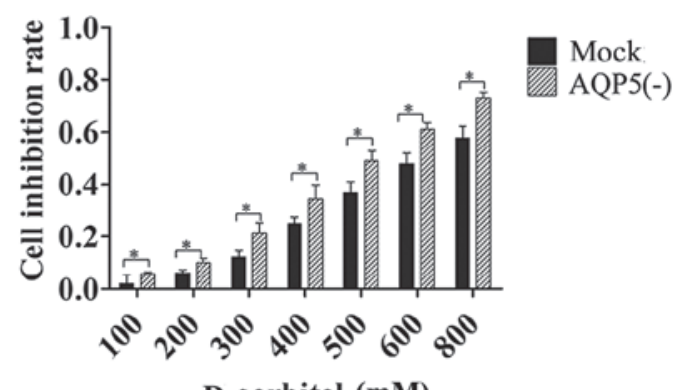

D-sorbitol (mM)

Figure 3. AQP5 was silenced by siRNA. The effects of AQP5 knockdown were examined by (A) RT-qPCR and (B) western blotting. GAPDH was used as an intrinsic control. (C) Cell inhibition rates induced by hypertonic D-sorbitol (100-800 mM) medium were compared by MTT assays of cell viability when AQP5 was knocked down in AO3 ovarian cancer cells. "P<0.05 for the AQP5(-) group vs. the mock group. AQP, aquaporin; AQP5(-), AQP5 silenced; siRNA, small interfering RNA; RT-qPCR, reverse transcription-quantitative chain reaction; GAPDH, glyceraldehyde 3-phosphate dehydrogenase; MTT, 3-(4,5-dimethylthiazol-2-yl)-2,5-diphenyltetrazolium bromide.

A

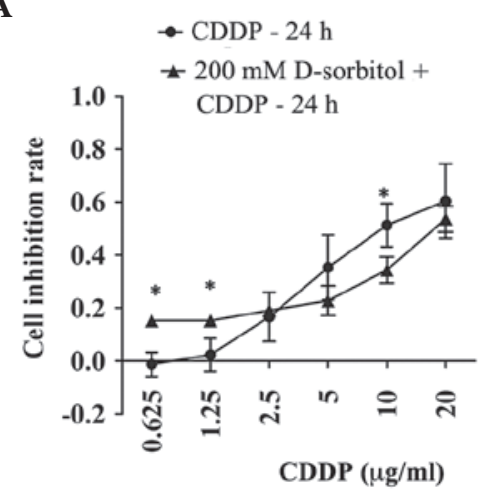

D

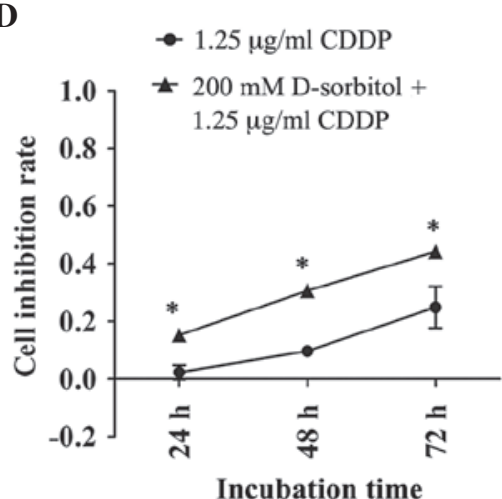

B

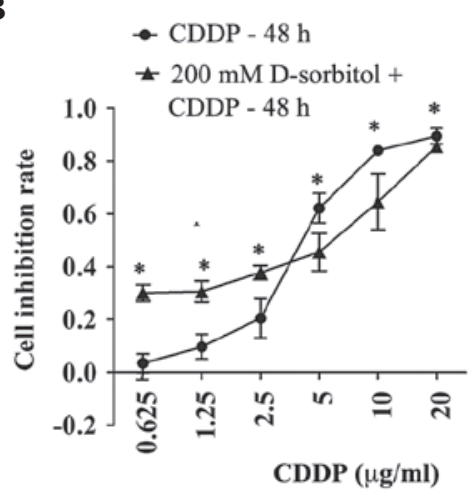

$\mathbf{E}$

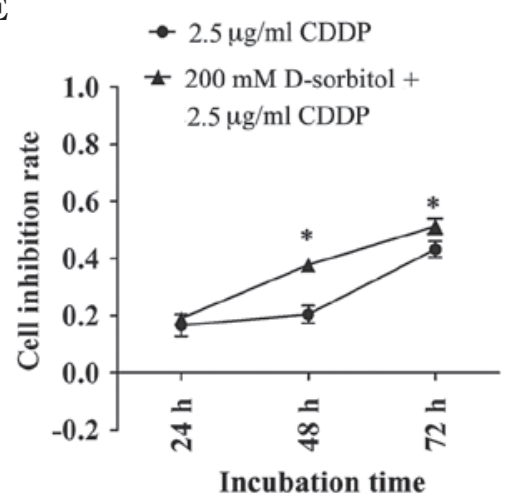

$\mathbf{C}$

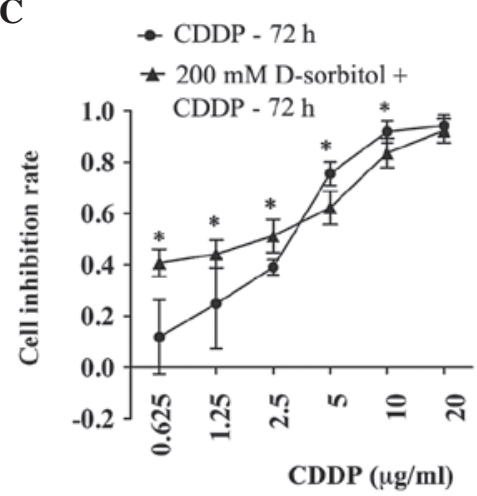

$\mathbf{F}$

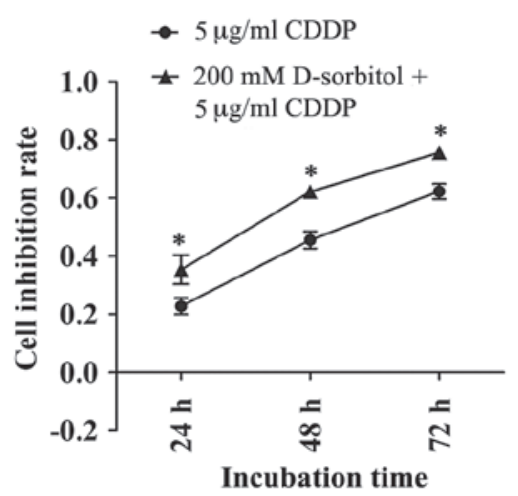

Figure 4. Changes of sensitivity to CDDP caused by extracellular hyperosmotic stress. 3AO cells were incubated with different concentrations of CDDP $(0.625-20 \mu \mathrm{g} / \mathrm{ml})$ with normal medium or hypertonic medium (200 mM D-sorbitol) for (A) $24 \mathrm{~h}$, (B) $48 \mathrm{~h}$, and (C) $72 \mathrm{~h}$ and the cell inhibition rates were compared by MTT assay. Sensitivity to CDDP at concentrations of (D) $1.25 \mu \mathrm{g} / \mathrm{ml}$, (E) $2.5 \mu \mathrm{g} / \mathrm{ml}$ and (F) $5 \mu \mathrm{g} / \mathrm{ml}$ was decreased in hypertonic medium (200 mM D-sorbitol) compared with that in isotonic medium, regardless of whether the incubation time was 24,48 or $72 \mathrm{~h}$. " $\mathrm{P}<0.05 \mathrm{CDDP}$ vs. D-sorbitol + CDDP. CDDP, cisplatin; MTT, 3-(4,5-dimethylthiazol-2-yl)-2,5-diphenyltetrazolium bromide.

hypertonic medium. Moreover, CDDP-induced cell death had dose- and time-dependent effects that were independent of a hypertonic or isotonic extracellular environment.

Effect of hyperosmotic stress on sensitivity to CDDP is associated with AQP5 expression in ovarian tumors. To examine the effect of CDDP on AQP5 expression under conditions of hyperosmotic stress, hyperosmosis was induced by adding $200 \mathrm{mM}$ D-sorbitol to the normal culture medium and treating $3 \mathrm{AO}$ cells with increasing concentrations of CDDP $(1.25-10 \mu \mathrm{g} / \mathrm{ml})$ in hypertonic or isotonic medium for $24 \mathrm{~h}$. The RT-qPCR results demonstrated that CDDP had similar 
A

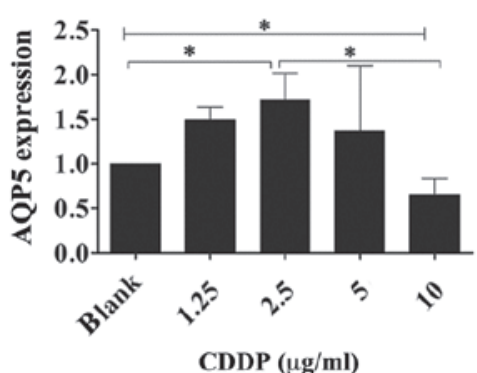

D

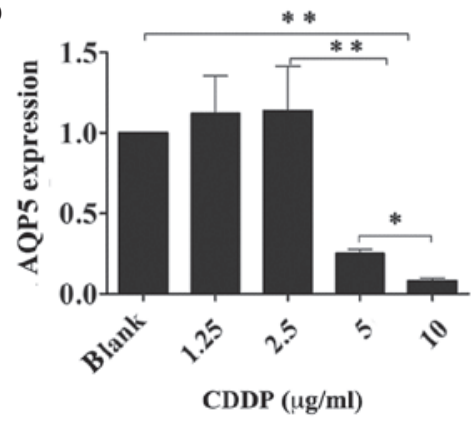

B

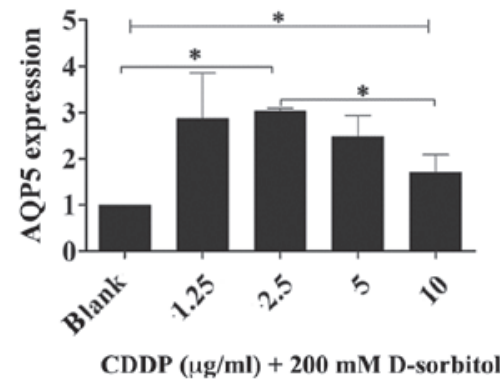

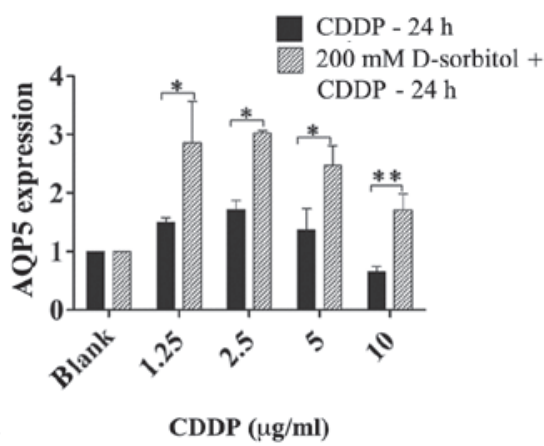

$\mathbf{E}$

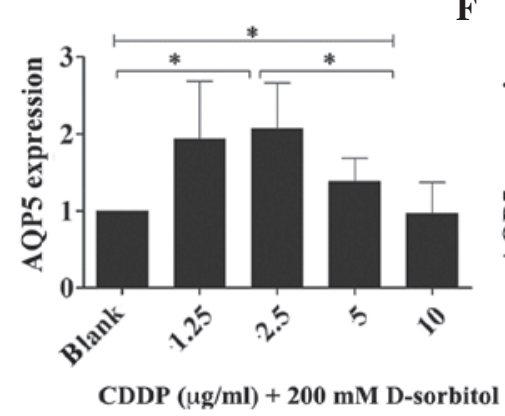

CDDP - $48 \mathrm{~h}$

$200 \mathrm{mM}$ D-sorbitol + CDDP $-48 \mathrm{~h}$
G

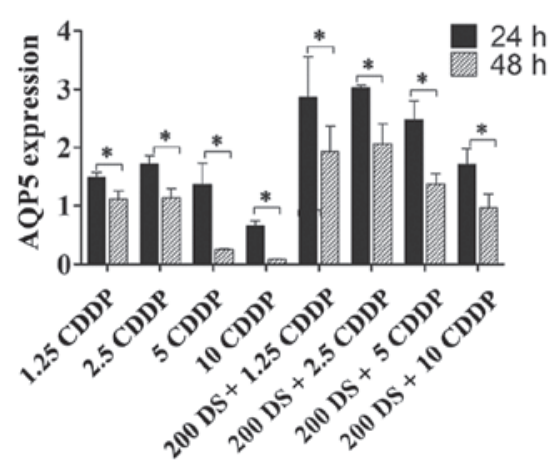

H
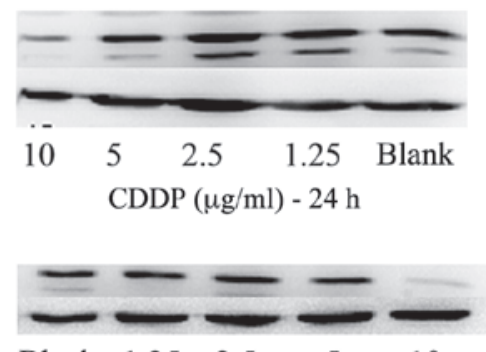

AQP5 (28 kDa)

GAPDH (37 kDa)

AQP5 (28 kDa)

GAPDH (37 kDa)

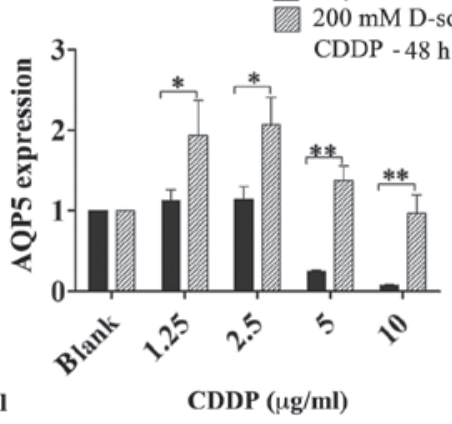

$\begin{array}{lllll}\text { Blank } & 1.25 & 2.5 & 5 & 10\end{array}$

$\operatorname{CDDP}(\mu \mathrm{g} / \mathrm{ml})-48 \mathrm{~h}$

Figure 5. AQP5 expression in response to CDDP in hypertonic and isotonic medium in 3AO cells. (A-C) AQP5 expression levels were measured by RT-qPCR when $3 \mathrm{AO}$ cells were treated with CDDP in (A) regular medium or (B) $200 \mathrm{mM}$ D-sorbitol medium for $24 \mathrm{~h}$ and (C) AQP5 expression levels under the two osmotic conditions were compared. (D-F) treatment time was prolonged to $48 \mathrm{~h}$; AQP5 mRNA expression following treatment with CDDP in (D) isotonic medium or (E) hypertonic medium (200 mM D-sorbitol) and (F) the comparison between them as determined by RT-qPCR. (G) AQP5 mRNA expression levels were decreased by CDDP (expressed in units of $\mu \mathrm{g} / \mathrm{ml}$ ) in regular medium and hypertonic medium including $200 \mathrm{mM}$ D-sorbitol (200 DS) as the treatment time was extended. (H) AQP5 protein expression levels following treatment with CDDP $(0.625-20 \mu \mathrm{g} / \mathrm{ml})$ in regular medium for 24 and $48 \mathrm{~h}$ were measured by western blotting. ${ }^{*} \mathrm{P}<0.05,{ }^{* *} \mathrm{P}<0.01$. AQP, aquaporin; CDDP, cisplatin; RT-qPCR, reverse transcription-quantitative chain reaction; GAPDH, glyceraldehyde 3-phosphate dehydrogenase.

effects on AQP5 mRNA expression in hypertonic or isotonic external environments (Fig. 5A-F). Following a 24-h incubation, a low-dose of CDDP $(<2.5 \mu \mathrm{g} / \mathrm{ml})$ enhanced AQP5 mRNA expression under hypertonic and isotonic extracellular conditions. At CDDP concentrations $\geq 5 \mu \mathrm{g} / \mathrm{ml}$, AQP5 expression was inversely proportional to the CDDP dose, and was reduced compared with that of $2.5 \mu \mathrm{g} / \mathrm{ml}$ CDDP (Fig. 5A and B). After $48 \mathrm{~h}$ of incubation, $\geq 5 \mu \mathrm{g} / \mathrm{ml}$ CDDP attenuated AQP5 expression levels by a large margin in an isotonic environment when compared with those in the blank cells or the cells treated with $2.5 \mu \mathrm{g} / \mathrm{ml}$ CDDP (Fig. 5D). However, when cells were treated with CDDP in hypertonic medium for $48 \mathrm{~h}$ different consequences were observed, with CDDP at doses $\leq 2.5 \mu \mathrm{g} / \mathrm{ml}$ inducing intensified AQP5 mRNA expression levels, while AQP5 mRNA expression levels were reduced at CDDP concentrations $\geq 5 \mu \mathrm{g} / \mathrm{ml}$ compared with those observed for $2.5 \mu \mathrm{g} / \mathrm{ml} \mathrm{CDDP} \mathrm{(Fig.} \mathrm{5E).} \mathrm{Furthermore,} \mathrm{as} \mathrm{the} \mathrm{incubation}$ time was prolonged, AQP5 expression levels were reduced by CDDP, regardless of whether the extracellular medium was hypertonic or isotonic (Fig. 5G), and the changes in AQP5 protein expression in response to CDDP were synchronized with those of AQP5 mRNA (Fig. 5H).

Nevertheless, the primary distinction between the two kinds of extracellular medium was that hyperosmosis enhanced the expression level of AQP5 mRNA at every dose of CDDP tested compared with those in isotonic medium, regardless of 
whether the incubation time was prolonged (48 h) or not (24 h) (Fig. 5C and F).

\section{Discussion}

Members of the AQP family have been associated with several types of tumors and can affect cell migration, proliferation, and angiogenesis (9). AQPs allow water to rapidly penetrate the cell membrane and are the primary determinants of membrane permeability to water. There is evidence that osmotic pressure can modify the expression of AQPs. In MLE-15 mouse lung epithelial cells, AQP5 was induced by hypertonic sorbitol-containing medium $(22,23)$, while in human airway epithelial cells, it was induced by hyperosmotic stress (24). AQP5 abundance decreased in a dose-dependent manner when MLE-12 mouse lung epithelial cells were exposed to hypotonic medium (25). In human keratinocytes, AQP3 mRNA expression was increased by hypertonic sorbitol-containing medium; however, AQP1, AQP4 and AQP9 mRNA expression remained unchanged (26). Moreover, hypertonicity promoted AQP2 expression in mouse principal kidney cortical collecting duct cells (27), while hypotonicity reduced it by attenuating cAMP-induced AQP2 promoter activity, a process mediated by TonE-mediated c-Jun N-terminal kinase activation (28). In mouse brain tissue, a $3 \% \mathrm{NaCl}$ hypertonic saline solution inhibited AQP4 mRNA and protein expression in astrocytes (29). Despite these results, to the best of our knowledge, AQP expression in response to osmotic stress has not been reported in ovarian cancer.

A previous study reported that AQP1, AQP5 and AQP9 expression levels were significantly increased in malignant ovarian cancer (10). In the present study, the effect of hypertonic sorbitol-containing medium on the proliferation of ovarian cancer cells and expression of AQP1, AQP3, AQP5, and AQP9 was determined. The results confirmed that ovarian cancer cell proliferation was dose- and time-dependently inhibited by a hypertonic extracellular environment, and that AQP5 mRNA and protein expression levels were induced by hypertonic stress. This provides the first evidence that AQP mRNA can be induced by hypertonic pressure in epithelial ovarian carcinoma, which is consistent with results in airway epithelial cells (22-24). Reduced induction of AQP5 by 300 or $400 \mathrm{mM}$ D-sorbitol-containing medium after $24 \mathrm{~h}$ could be associated with diminished cell viability at that level of osmotic stress (22). However, in the present study, a greater increase in AQP5 mRNA expression was observed with $400 \mathrm{mM}$ sorbitol medium after $48 \mathrm{~h}$. We propose that AQP5 expression can be regulated by osmotic pressure and is associated with cell viability, and the enhancing effects of hypertonic D-sorbitol on AQP5 expression were particularly evident when the incubation time was $48 \mathrm{~h}$.

Previous studies have determined that AQPs are important during membrane osmosis (30-32). In the current study, the MTT assay results showed that ovarian cancer cells were more susceptible to hypertonic medium after AQP5 expression was reduced by siRNA knockdown. The fact that AQP5 knockdown can reduce both osmotic water permeability and regulatory volume in human lung adenocarcinoma cells (30) may explain the effects observed in AQP5-knockdown ovarian cancer cells in response to hyperosmotic pressure. This indi- cates that AQP5 has an important role in osmotic homeostasis and that hypertonic stress is able to regulate AQP5 expression in ovarian cancer. Therefore, we speculate that ovarian cancer cells adapt to hypertonic pressure based on changes in AQP5 expression, since water permeability is affected by increased AQP5 expression, which allowed the cells to adapt to the extracellular hypertonic state.

The results of the present study also indicated that a D-sorbitol-mediated extracellular hypertonic environment can modify AQP1, AQP3, and AQP9 mRNA expression; however, the changes were not determined to be statistically significant. There are several factors that might explain this result: i) AQP1 is expressed primarily in the microvascular endothelium in ovarian cancer $(10,11)$; ii) AQP3 and AQP9 are aquaglyceroporins permeable to glycerol, urea, other small non-electrolytes and water (8); iii) AQP1 and AQP9 are expressed at low levels in $3 \mathrm{AO}$ cells (Fig. 2); and iv) AQPs respond differently to osmotic pressure in various tissues (22-29).

Our previous study revealed that AQP5 expression was decreased by CDDP in the CAOV3 cell line (15), which correlates with the present study, and chemosensitivity was influenced by AQPs in the SKOV3 cell line (21). The present study sought to determine the associations among CDDP sensitivity, AQP expression and osmotic pressure in the $3 \mathrm{AO}$ cell line. The influence of hyperosmotic pressure on sensitivity to CDDP and its association with the expression of AQP5 is clarified by the current results.

Resistance to CDDP is affected by many factors, including changes in drug uptake and efflux, increased drug metabolism in tumor cells, and DNA repair. Reduced uptake and enhanced efflux of drugs from cells mediated by membrane transporters and ion channels play an important role in drug sensitivity and resistance. Osmotic stress controls water influx and efflux across cells and may have an impact on drug metabolism to further affect drug sensitivity. However, there are few reports that have investigated this mechanism. We have previously demonstrated that hyperosmotic stress induced by sorbitol increases the sensitivity of SKOV3 cells to CDDP (21). The present study revealed that sensitivity to CDDP was modified by hypertonic pressure in the $3 \mathrm{AO}$ cell line. Moreover, AQP5 expression was modified significantly by hypertonic sorbitol medium and was essential for the response of ovarian cancer cells to extracellular hypertonic medium.

Our results indicate that $3 \mathrm{AO}$ cell sensitivity to CDDP is enhanced by extracellular hyperosmosis when the CDDP concentration is low, which may contribute to an induction of CDDP-mediated AQP5 expression, further increased by hypertonic stress. In addition, inhibition of cell proliferation at a high CDDP dose was decreased in hyperosmotic medium compared with that in isotonic medium, and this could be attributed to the downregulation of AQP5 expression caused by a high-dose CDDP being antagonized by hypertonic pressure. Accordingly, we hypothesized that the changes in sensitivity to CDDP induced by hypertonic medium were caused by an increase in AQP5 expression. In addition, the abnormal expression of AQP3 or AQP9 affects chemoresistance to arsenite in melanoma cells, lung cancer, primary cultured chorion and amnion cells (18-20), and sensitivity to CDDP is associated with AQPs in the SKOV3 cell line (21). On the basis of the present study, sensitivity to CDDP is closely associated 
with AQP5 expression in ovarian cancer. However, additional studies are necessary to determine the association between CDDP sensitivity and AQP5 expression and to elaborate on the regulatory mechanism involved.

In summary, the present study demonstrated that extracellular hypertonic stress inhibits the proliferation of $3 \mathrm{AO}$ cells, and that increased expression of AQP5 plays an important role in the response of ovarian cancer cells to hypertonic medium, which regulates CDDP sensitivity in ovarian cancer. Changes in CDDP sensitivity induced by hyperosmosis were found to be associated with changes in AQP5 expression, indicating that AQP5 expression is relevant to CDDP sensitivity. The results show that CDDP sensitivity was affected by extracellular hyperosmosis in an ovarian cancer cell line, which suggests a novel direction for ovarian cancer research. In addition, the important role of AQP5 expression in the regulation of osmotic pressure and sensitivity to chemotherapy suggest it may be a new focus for ovarian cancer-targeted therapy.

\section{Acknowledgements}

This study was supported by a grant from the National Natural Science Foundation of China (grant no. 81202064).

\section{References}

1. Wiedemeyer WR, Beach JA and Karlan BY: Reversing platinum resistance in high-grade serous ovarian carcinoma: Targeting BRCA and the homologous recombination system. Front Oncol 4: 34, 2014.

2. Bogliolo S, Cassani C, Gardella B, Musacchi V, Babilonti L, Venturini PL, et al. Oxaliplatin for the treatment of ovarian cancer. Expert opinion on investigational drugs. 2015;24(9):1275-86.

3. Benga G: Water channel proteins (later called aquaporins) and relatives: Past, present and future. IUBMB Life 61: 112-133, 2009.

4. Gomes D, Agasse A, Thiébaud P, Delrot S, Gerós H and Chaumont F: Aquaporins are multifunctional water and solute transporters highly divergent in living organisms. Biochim Biophys Acta 1788: 1213-1228, 2009.

5. Agre P and Kozono D: Aquaporin water channels: Molecular mechanisms for human diseases. FEBS Lett 555: 72-78, 2003.

6. Nozaki K, Ishii D and Ishibashi K: Intracellular aquaporins: Clues for intracellular water transport? Pflugers Arch 456: 701-707, 2008

7. Zardoya R: Phylogeny and evolution of the major intrinsic protein family. Biol Cell 97: 397-414, 2005.

8. Zhang D, Tan YJ, Qu F, Sheng JZ and Huang HF: Functions of water channels in male and female reproductive systems. Mol Aspects Med 33: 676-690, 2012.

9. Ribatti D, Ranieri G, Annese T and Nico B: Aquaporins in cancer. Biochim Biophys Acta 1840: 1550-1553, 2014.

10. Yang JH, Yu YQ and Yan CX: Localisation and expression of aquaporin subtypes in epithelial ovarian tumours. Histol Histopathol 26: 1197-1205, 2011.

11. Takal MK, Baykal C, Başer E, Kaya MD, Dursun P, Ozen O, Haberal AN and Ayhan A: Does Aquaporin-1 expression have clinical significance in serous epithelial ovarian cancer? J Turk Ger Gynecol Assoc 14: 130-135, 2013.

12. Yang JH, Shi YF, Cheng Q and Deng L: Expression and localization of aquaporin-5 in the epithelial ovarian tumors. Gynecol Oncol 100: 294-299, 2006.

13. Yang JH, Shi YF, Chen XD and Qi WJ: The influence of aquaporin-1 and microvessel density on ovarian carcinogenesis and ascites formation. Int J Gynecol Cancer 16 (Suppl 1): 400-405, 2006.
14. Ji C, Cao C, Lu S, Kivlin R, Amaral A, Kouttab N, Yang H, Chu W, Bi Z, Di W and Wan Y: Curcumin attenuates EGF-induced AQP3 up-regulation and cell migration in human ovarian cancer cells. Cancer Chemother Pharmacol 62: 857-865, 2008.

15. Yang J, Yan C, Zheng W and Chen X: Proliferation inhibition of cisplatin and aquaporin 5 expression in human ovarian cancer cell CAOV3. Arch Gynecol Obstet 285: 239-245, 2012.

16. Yan C, Yang J, Shen L and Chen X: Inhibitory effect of Epigallocatechin gallate on ovarian cancer cell proliferation associated with aquaporin 5 expression. Arch Gynecol Obstet 285: 459-467, 2012.

17. Day RE, Kitchen P, Owen DS, Bland C, Marshall L, Conner AC, Bill RM and Conner MT: Human aquaporins: Regulators of transcellular water flow. Biochim Biophys Acta: 1492-1506, $1840,2014$.

18. Gao L, Gao Y, Li X, Howell P, Kumar R, Su X, Vlassov AV, Piazza GA, Riker AI, Sun D and Xi Y: Aquaporins mediate the chemoresistance of human melanoma cells to arsenite. Mol Oncol 6: 81-87, 2012.

19. Miao ZF, Chang EE, Tsai FY, Yeh SC, Wu CF, Wu KY, Wang CJ and Tsou TC: Increased aquaglyceroporin 9 expression disrupts arsenic resistance in human lung cancer cells. Toxicol In Vitro 23: 209-216, 2009.

20. Yoshino Y, Yuan B, Kaise T, Takeichi M, Tanaka S, Hirano T, Kroetz DL and Toyoda H: Contribution of aquaporin 9 and multidrug resistance-associated protein 2 to differential sensitivity to arsenite between primary cultured chorion and amnion cells prepared from human fetal membranes. Toxicol Appl Pharmacol 257: 198-208, 2011.

21. Chen XJ, Chen WM, Ding XY, Zheng W, Zhang Q and Yang JH: Effects of aquaporins on chemosensitivity to cisplatin in ovarian cancer cells. Arch Gynecol Obstet 290: 525-532, 2014.

22. Hoffert JD: Hypertonic induction of aquaporin-5 expression through an ERK-dependent pathway. J Biol Chem 275: 9070-9077, 2000.

23. Zhou B, Ann DK, Li X, Kim KJ, Lin H, Minoo P, Crandall ED and Borok Z: Hypertonic induction of aquaporin-5: Novel role of hypoxia-inducible factor-1alpha. Am J Physiol Cell Physiol 292: C1280-C1290, 2007.

24. Pedersen PS, Braunstein TH, Jørgensen A, Larsen PL, Holstein-Rathlou NH and Frederiksen O: Stimulation of aquaporin-5 and transepithelial water permeability in human airway epithelium by hyperosmotic stress. Pflugers Arch 453: 777-785, 2007.

25. Sidhaye VK, Güler AD, Schweitzer KS, D'Alessio F, Caterina MJ and King LS: Transient receptor potential vanilloid 4 regulates aquaporin-5 abundance under hypotonic conditions. Proc Natl Acad Sci USA 103: 4747-4752, 2006.

26. Sugiyama Y, Ota Y, Hara M and Inoue S: Osmotic stress up-regulates aquaporin-3 gene expression in cultured human keratinocytes. Biochim Biophys Acta 1522: 82-88, 2001.

27. Li SZ, McDill BW, Kovach PA, Ding L, Go WY, Ho SN and Chen F: Calcineurin-NFATc signaling pathway regulates AQP2 expression in response to calcium signals and osmotic stress. Am J Physiol Cell Physiol 292: C1606-C1616, 2006.

28. Saito T, Saito T, Kasono K, Tamemoto H, Kawakami M, Sasaki S and Ishikawa SE: Hypotonicity reduces the activity of murine aquaporin-2 promoter induced by dibutyryl cAMP. Exp Physiol 93: 1147-1156, 2008.

29. Cao C, Yu X, Liao Z, Zhu N, Huo H, Wang M, Ji G, She H, Luo Z and Yue S: Hypertonic saline reduces lipopolysaccharide-induced mouse brain edema through inhibiting aquaporin 4 expression. Crit Care 16: R186, 2012.

30. Chen Z, Zhang Z, Gu Y and Bai C: Impaired migration and cell volume regulation in aquaporin 5-deficient SPC-A1 cells. Respir Physiol Neurobiol 176: 110-117, 2011.

31. Solenov E, Watanabe H, Manley GT and Verkman AS: Sevenfold-reduced osmotic water permeability in primary astrocyte cultures from AQP-4-deficient mice, measured by a fluorescence quenching method. Am J Physiol Cell Physiol 286: C426-C432, 2004.

32. Thiagarajah JR and Verkman AS: Aquaporin deletion in mice reduces corneal water permeability and delays restoration of transparency after swelling. J Biol Chem 277: 19139-19144, 2002. 